\title{
Element-Specific Density Profiles in Interacting Biomembrane Models
}

\author{
Emanuel Schneck ${ }^{\mathrm{a},{ }^{*}}$, Ignacio Rodriguez-Loureiro ${ }^{\mathrm{a}}$, Luca Bertinetti ${ }^{\mathrm{a}}$, Egor Marin ${ }^{\mathrm{b}}$, Dmitri Novikov $^{\mathrm{c}}$, \\ Oleg Konovalov ${ }^{\mathrm{d}}$, and Georgi Gochev ${ }^{\mathrm{a}, \mathrm{e}}$. \\ a Max Planck Institute of Colloids and Interfaces, Am Mühlenberg 1, 14476 Potsdam, Germany \\ ${ }^{\mathrm{b}}$ Moscow Institute of Physics and Technology (MIPT), 141700 Dolgoprudniy, Russia \\ 'Deutsches Elektronen-Synchrotron (DESY), 22607 Hamburg, Germany \\ ${ }^{d}$ European Synchrotron Radiation Facility, 71 avenue des Martyrs, 38000 Grenoble, France \\ 'Institute of Physical Chemistry, Bulgarian Academy of Sciences, 1113 Sofia, Bulgaria \\ *Corresponding author: schneck@mpikg.mpg.de, Phone: +49-331567-9404, Fax: +49-331567-9402
}

Keywords: surfaces, interfaces, lipid membranes, x-ray scattering, polymer brushes

\begin{abstract}
Surface interactions involving biomembranes, such as cell-cell interactions or membrane contacts inside cells play important roles in numerous biological processes. Structural insight into the interacting surfaces is a prerequisite to understand the interaction characteristics as well as the underlying physical mechanisms. Here, we work with simplified planar experimental models of membrane surfaces, composed of lipids and lipopolymers. Their interaction is quantified in terms of pressure-distance curves using ellipsometry at controlled dehydrating (interaction) pressures. For selected pressures, their internal structure is investigated by standing-wave $x$-ray fluorescence (SWXF). This technique yields specific density profiles of the chemical elements $\mathrm{P}$ and $\mathrm{S}$ belonging to lipid headgroups and polymer chains, as well as counter-ion profiles for charged surfaces.
\end{abstract}

\section{Introduction}

Nanometric layers formed by molecular assemblies in two-dimensional architectures are major components of soft and biological matter [1,2]. Their structural organization and biological or technological functions are highly sensitive to the mutual interactions of their surfaces in the aqueous environment [1,3]. Important examples in biology are the membranes of cells and organelles, which are typically found under rather crowded conditions [4], so that membranemembrane interactions play a key role for their functions. The character of the interaction in terms of magnitude and range, as well as whether it is mainly attractive or repulsive, affects cell adhesion $[5,6]$, vesicle release, the spontaneous formation of membrane stacks [7] and the properties of bacterial biofilms [8]. It in general involves spatially extended (generic) and specific ligand-receptor contributions and is determined by the chemical composition of the membrane surfaces comprising neutral and charged lipid head groups and membrane-bound saccharides, polypeptides, and macromolecules. Similarly, the interaction between technological interfaces also plays an important role in a multitude of applications such as liquid purification and separation chemistry [9], or lubrication [10]. Liquid/liquid interfaces, for example, are commonly stabilized by self-assembled amphiphilic molecules including surfactants, proteins, and polymers, with the aim of rendering their mutual interaction predominantly repulsive thus providing stability of ("soft") colloids [11]. 
The interaction between two surfaces across water is typically described in terms of pressuredistance curves, which relate the interaction (or: disjoining) pressure $\Pi$ to the surface separation or (equivalent) water layer thickness $D_{\mathrm{w}}$. For fixed temperature $T$ and ambient pressure $p, \Pi$ represents the derivative of the Gibbs free energy $G$ per unit area $A$ with respect to $D_{\mathrm{w}}[12]$,

$\Pi\left(D_{w}\right)=-\frac{1}{A}\left(\frac{d G}{d D_{w}}\right)_{T, p}$.

Pressure-distance relations are commonly determined by subjecting multilamellar stacks of interacting surfaces to dehydrating pressures of known magnitude [13]. So-called equivalent pressures can be exerted either by bringing the multilayers into contact with osmotically active polymer solutions, or by controlling the ambient relative humidity $h_{\text {rel, }}$ in which case

$\Pi\left(h_{\text {rel }}\right)=-\frac{k_{B} T}{v_{w}} \ln \left(h_{\text {rel }}\right)$,

where $v_{\mathrm{w}}$ denotes the volume of a water molecule and $k_{\mathrm{B}}$ is the Boltzmann constant. For biologically or technologically relevant "soft" interfaces, the interaction characteristics are often closely related to molecular conformations and charge distributions [3]. For instance, forces between surfaces displaying charged chemical moieties depend on the exact location of the charges and on their ability to spatially rearrange upon the surfaces' mutual approach. Similarly, electrostatic interactions between charged surfaces are sensitive to the distribution of counterions on the axis perpendicular to the surface plane [14]. Polymer-decorated surfaces, on the other hand, interact differently if the opposing polymer layers are able to overlap [15]. In other words, structural aspects like molecular conformations or element distributions are indicative of the interaction mechanisms and thus of great relevance. However, insight into structures "buried" between two surfaces is difficult to obtain experimentally. $\mathrm{X}$-ray and neutron scattering are among the very few techniques that can probe such structures with the required sub-nanometer spatial resolution. Specular reflectometry reveals matter density profiles perpendicular to an interface $[16,17]$. The latter can then be interpreted in terms of interfacial distributions of chemical components $[18,19]$. This approach has been taken also to investigate the structure of lipid layers incorporating lipopolymers [20,21] and of interacting soft interfaces [22-24]. However, it is not always possible to deduce the relevant structural features from such "global" density profiles. In contrast, x-ray fluorescence allows determining element-specific density profiles across an interface [25]. The technique is based on the characteristic fluorescence induced by the illuminating $\mathrm{x}$-rays via photoelectric ionization and has commonly been used to study element distributions at gas/liquid interfaces [26-28]. Ångstrom resolution perpendicular to the interface can be achieved with $x$-ray fluorescence under Bragg diffraction or mirror reflection conditions. In particular, planar nanometer-thick multilayers allow to create strongly modulated standing $x$-ray waves above the multilayer surface, whose period can be adjusted to match the typical length scales of the objects investigated $[29,30]$. Such standing-wave $x$-ray fluorescence (SWXF) studies have so far dealt with the fluorescence of comparatively heavy elements, often used as labels [25]. Only recently we have established a label-free implementation of SWXF, which is suited to directly localize lighter and biologically relevant chemical elements like $P$ and $S$ with atom scale precision [31]. 
In the present work, we utilize this technique for the label-free element-specific structural investigation of interacting layers approximately mimicking the surfaces of distinct types of biological membranes. Measurements were carried out at varying humidity corresponding to different equivalent interaction pressures and surface separations. The results are discussed with respect to the pressure-distance curves of the same systems, which were determined in the present work by ellipsometry.

a
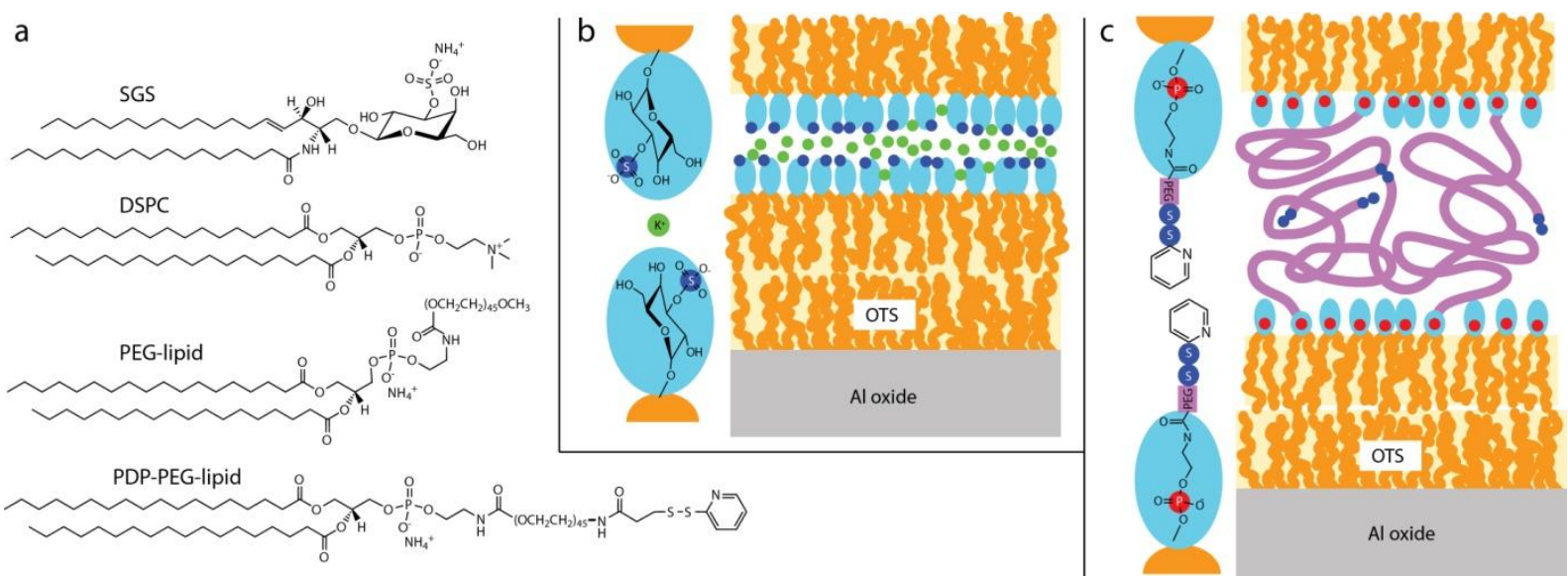

Figure 1: (a) Chemical structures of the amphiphilic molecules SGS (3-O-sulfo-D-galactosyl-ß1-1'-Nheptadecanoyl-D-erythro-sphingosine), DSPC (1,2-distearoyl-sn-glycero-3-phosphocholine), PEG-lipid (1,2distearoyl-sn-glycero-3-phosphoethanolamine-N-[methoxy(polyethylene glycol)-2000]), and PDP-PEG-lipid (1,2distearoyl-sn-glycero-3-phosphoethanolamine-N-[PDP(polyethylene glycol)-2000]). (b and c) Schematic illustration of the double-monolayer samples investigated in the present study. (b) Interacting SGS surfaces bearing $\mathrm{S}$ atoms (dark blue dots) in the lipid headgroups. Green dots indicate $\mathrm{K}^{+}$counter ions. (c) Interacting lipid-anchored PEG brushes bearing $P$ atoms (red dots) in the lipid headgroups and $S$ atoms at the end of the PEG chains. The solid surfaces are hydrophobically functionalized with octadecyltrichlorosilane (OTS).

\section{Results}

Fig. $1 \mathrm{~b}$ and c schematically illustrate the studied double monolayer samples composed of the amphiphilic molecules shown in Fig. 1 a. They act as defined models of interacting membrane surfaces with known composition. Interacting surfaces composed of the glycolipid (SGS) (Fig. 1 b) represent interacting membranes that contain significant densities of negatively charged sulfoglycolipids, like photosynthetic membranes [32]. Each SGS molecule (Fig. 1 a) comprises one $S$ atom that can be targeted by SWXF. These highly charged models of membrane surfaces are also suited to study the behavior of counterions. Here, the samples were prepared such that the counterions are $\mathrm{K}^{+}$ions (see Methods section) that can also be localized with the SWXF settings employed. Interacting lipid-anchored PEG brushes (formed by 10 mol\% lipopolymers anchored into a DSPC matrix, see Fig. 1c) in a generic way represent interacting membrane surfaces displaying hydrophilic macromolecules, such as cell glycocalyx [33], or the outer LPS surfaces of Gram-negative bacteria [8]. SWXF allows localizing the P atoms in DSPC and the lipopolymers (PEG-lipid or PDP-PEGlipid) and the $S$ atoms in the labeled polymer endpoints of PDP-PEG-lipid (Fig. 1 a). With that, this system is uniquely suited for the simultaneous localization of the grafting surface (via its $\mathrm{P}$ content) 
and the endpoints of the brush (via its S content). Such architectures are therefore interesting also in context with a validation of theoretical models describing the conformation of interacting polymer brushes [34].

Double-monolayer architectures like the ones illustrated in Fig. $1 \mathrm{~b}$ and c have similarities with commonly studied supported membranes (the solid support [35]) and lipid monolayers at the air/water interface (the vapor phase [20]). Importantly, they enable the controlled interaction of well-defined surfaces and the simultaneous hydration-dependent structural investigation. Since the amphiphilic monolayers are flexible, a homogeneous surface separation on the planar substrate is always realized, even if impurities, for example dust particles, locally perturb the interaction. This is in contrast to approaches involving two planar solids, where creation of a defined interaction distance is generally challenging [36]. Moreover, double monolayers can be used also for the study of non-symmetrical interaction scenarios and for molecular compositions that do not lead to the spontaneous formation of lamellar phases. This is in contrast to the classical multilayer swelling approach [37]. The interaction strength can be quantified in terms of the equivalent interaction pressure, which is dictated by the humidity (see Eq. 2). The absence of bulk water serves for the controlled dehydration and is therefore desired. For high humidities close to saturation ( $h_{r e l} \rightarrow 100 \%$ ), the samples are highly swollen and comprise a large amount of water corresponding

to a large equivalent water layer thickness $D_{w}=v_{w} N_{w} / A$, where $N_{\mathrm{w}}$ is the number of water molecules per unit area $A$. Under this condition the surfaces are further apart than for low humidities, where the samples are less swollen and $D_{\mathrm{w}}$ is smaller.

\subsection{Pressure-distance curves}

Fig. 2 shows the pressure-distance curves of interacting SGS surfaces and lipid-anchored PEG brushes as determined by ellipsometry (see Methods section). For both systems the interaction pressures are repulsive, meaning that work has to be performed in order to reduce $D_{\mathrm{w}}$. Close to completely dehydrated conditions $\left(D_{w} \rightarrow 0\right.$ ) the pressures reach magnitudes of several kbars. Upon reducing the equivalent pressure (i.e., increasing the humidity) to the lowest levels achieved in the present work, both systems take up a substantial amount of water, $D_{\mathrm{w}}>2 \mathrm{~nm}$. But despite these similarities, the repulsion mechanisms leading to water uptake upon releasing the equivalent pressure have to be considered totally different: The repulsion between the negatively charged SGS surfaces is mainly of electrostatic nature. As was shown recently, the range of the repulsive force between glycolipid surfaces increases substantially when negatively charged sulfoglycolipids are incorporated [32]. In contrast, charges play only a minor role for the repulsion between the lipid-anchored PEG brushes. Instead, the hydration of the polymer chains, as well as steric forces related to their configurational entropy are more important. While pure DSPC surfaces like other phosphatidylcholine (PC) lipid surfaces merely experience short range hydration repulsion $[37,38]$, the incorporation of PEG-lipid was shown to induce additional steric repulsion that systematically increases with polymer length and grafting density [39]. 


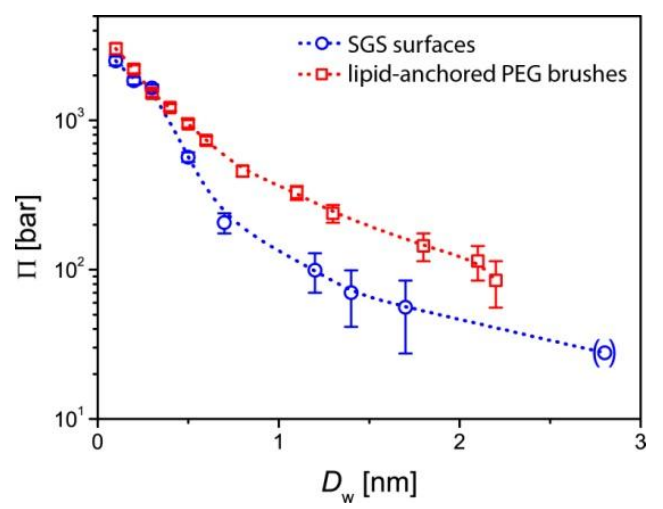

Figure 2: Pressure-distance curves of interacting SGS surfaces (circles) and lipid-anchored PEG brushes (squares). Error bars represent the uncertainty of the humidity sensor. For the data point in brackets the error in $\Pi$ is comparable to its value. Dashed lines serve to guide the eye.

\subsection{Element concentration profiles}

Fig. 3 a illustrates the geometry of the SWXF experiments. The technique is based on the elementcharacteristic fluorescence induced via photoelectric ionization by a standing $\mathrm{x}$-ray wave. The angle of incidence is denoted with $\theta$. Close to the Bragg angle $\theta_{\mathrm{B}}$ of the multilayer substrate (here: $\left.\theta_{\mathrm{B}}=1.13^{\circ}\right)$, strong reflection occurs, giving rise to a highly modulated standing wave (SW) intensity pattern. Fig. $3 \mathrm{~b}$ (symbols) shows the intensity of the reflected beam in a narrow interval of $\pm 0.08^{\circ}$ around $\theta_{\mathrm{B}}$. The solid line represents the theoretically modeled reflectivity based on a suitable layer representation of the electron density profile of the periodic $\mathrm{Al} / \mathrm{Ni}$ layers (see Methods section). The $\theta$-dependent fluorescence intensity of a target element $j, l_{j}(\theta)$, scales with a spatial integral containing the elemental concentration profile perpendicular to the interface, $c_{\mathrm{j}}(z)$, and the $\theta$ dependent SW intensity $\Phi(\theta, z)$ :

$I_{j}(\theta)=A \int_{-\infty}^{\infty} \Phi(\theta, z) c_{j}(z) d z$

where $A$ is a pre-factor that involves, among others, fluorescence yield, beam footprint, and detector field-of-view, and therefore weakly depends on $\theta$ in general [31]. According to Eq. 3, $c_{\mathrm{j}}(\mathrm{z})$ can thus be deduced from $l_{j}(\theta)$, if $\Phi(\theta, z)$ is known. The latter was computed via the phase-correct summation of all reflected and transmitted partial waves [28] from the layer representation mentioned above. For illustration, calculated SW intensity patterns $\Phi(\theta, z)$ above the solid surface are shown in Fig. $3 \mathrm{c}$ for $\theta=\theta_{\mathrm{B}}$ and for $\theta=\theta_{\mathrm{B}} \pm 0.02^{\circ}$ (see vertical lines in Fig. $3 \mathrm{~b}$ ). As $\theta$ is increased through the Bragg condition, the nodes and antinodes of the SW intensity shift towards the solid surface by approximately half a multilayer period. 


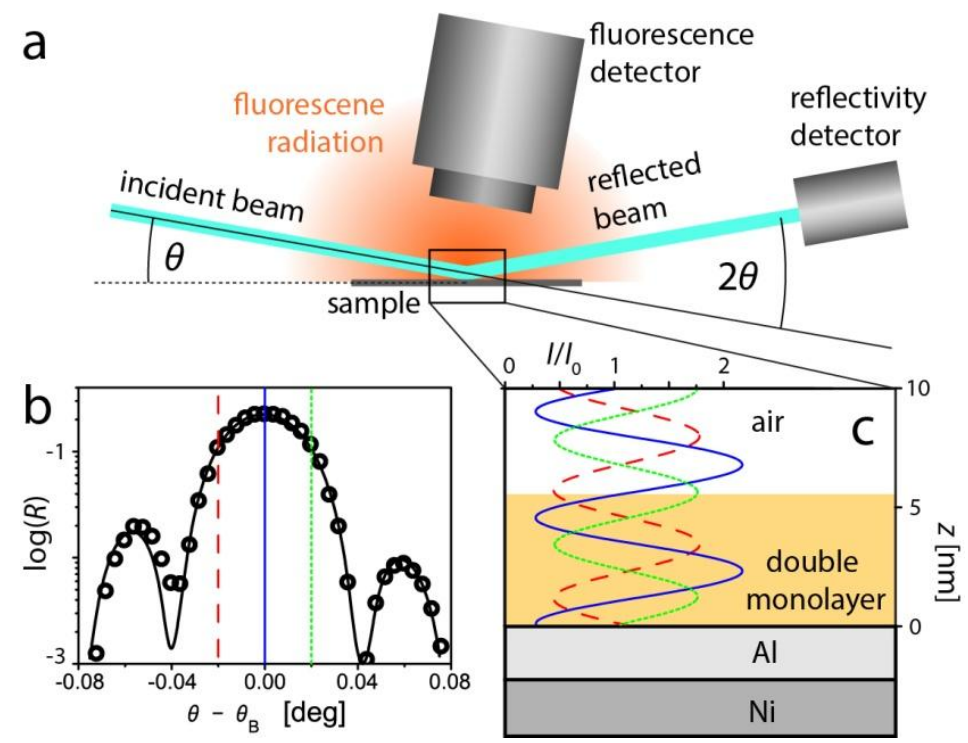

Figure 3: (a) Sketch of the SWXF measurement setup. Incident and reflected x-ray beams are indicated with bright blue lines. The isotropically emitted $x$-ray fluorescence radiation is indicated in red. The incident angle is denoted with $\theta$. (b) Measured reflectivity curve (symbols) in the vicinity of the first Bragg angle $\theta_{\mathrm{B}}$ of the solid multilayers and theoretical model (solid black line). (c) Standing wave intensity patterns $\Phi(\theta, z)$ above the solid surface computed for $\theta=\theta_{\mathrm{B}}$ (solid dark blue line) and for $\theta=\theta_{\mathrm{B}} \pm 0.02^{\circ}$ (dotted green and dashed red lines, respectively), corresponding to the vertical lines in panel $b$. The axis perpendicular to the planar solid surface is denoted with $z$. The region shaded in orange schematically represents the supported monolayer architectures.

Fluorescence spectra were measured for various incident angles in $\theta$-scans around the Bragg angle. Fig. 4 shows a representative spectrum of interacting lipid-anchored PEG brushes (see Fig. $1 \mathrm{c}$ ), recorded exemplarily for $\theta=\theta_{\mathrm{B}}$. The spectrum exhibits the characteristic fluorescence peaks of $\mathrm{P}$ and $S$ (see inset) together with peaks of $\mathrm{Al}$ and other chemical elements found in the multilayer substrates and in the sample environment ( $\mathrm{Ar}, \mathrm{Ti}$, and $\mathrm{Cr}$ ). Ni does not emit fluorescence because the incident beam energy is below the $\mathrm{K}$-shell edge of $\mathrm{Ni}$. The fluorescence intensities of the target elements were extracted from the spectra for each incident angle. For this purpose, from the sample spectra the corresponding reference spectra of the substrates in the absence of the interacting monolayers were subtracted. The peaks of the target elements in the resulting difference spectra were then modeled with Gaussian functions, and the obtained amplitudes were used to build for each element its $\theta$-dependent fluorescence intensity curves $I_{j}(\theta)$. In the last step, based on the known shape of $\Phi(\theta, z)$, the intensities curves were modeled according to Eq. 3 using a suitable parameterization of the element profiles $c_{j}(z)$. The weak angle-dependence of the pre-factor $A$ in the equation was approximated linearly with an adjustable parameter for the slope, as was done in ref [31]. 


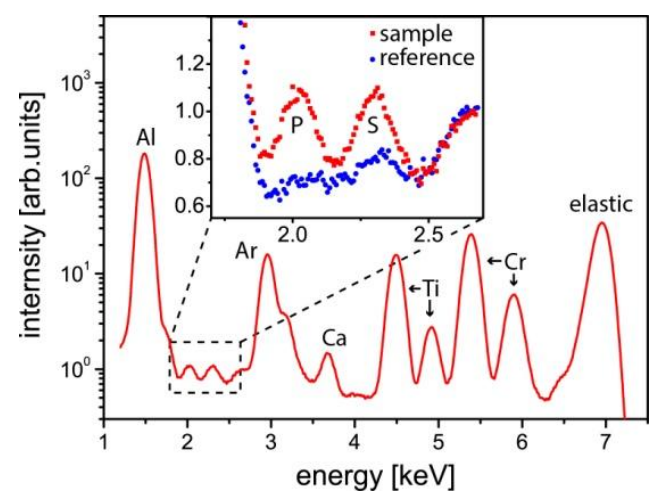

Figure 4: X-ray fluorescence spectrum of interacting lipid-anchored PEG brushes (see Fig. $1 \mathrm{c}$ ) recorded exemplarily for $\theta=\theta_{\mathrm{B}}$. The spectrum comprises characteristic peaks of $\mathrm{Al}$ and other chemical elements found in the multilayer substrates and in the sample environment ( $\mathrm{Ar}, \mathrm{Ti}$, and $\mathrm{Cr}$ ). Inset: Characteristic fluorescence peaks of $P$ and $S$. Unlike the main panel the inset also shows the comparison with the reference spectrum obtained in the absence of the $\mathrm{P}$ and $\mathrm{S}$ loaded double monolayer.

\subsubsection{Interacting SGS surfaces}

Fig. 5 a (bottom curve, symbols) shows the angle-dependent $S$ fluorescence, $I_{S}(\theta)$, of interacting SGS surfaces (Fig. 1 b) at a low humidity of $h_{\text {rel }} \approx 50 \%$, corresponding to $\Pi \approx 1$ kbar (Eq. 2). As suggested by the pressure distance curve (Fig. 2, circles), under strongly dehydrating conditions the water layer between the two sulfur-bearing surfaces is only few $\AA$ thick, comparable to the surface roughness. The $S$ distributions belonging to the two surfaces are therefore expected to strongly overlap, resulting in an overall $S$ distribution with only one maximum. The $S$ concentration profile $c_{S}(z)$ was accordingly modeled as a unimodal distribution,

$c_{S}(z)=c_{\max } e^{-\left(z-z_{S}\right)^{2} /\left(2 \sigma_{S}^{2}\right)}$.

In this Gaussian representation, the amplitude $c_{\max }$, the center position $z_{\mathrm{S}}$ and the width $\sigma_{\mathrm{s}}$ are adjustable fitting parameters. While $c_{\max }$ merely acts as a scaling parameter, $z_{\mathrm{S}}$ is linked to the angular positions of the extrema in $l_{\mathrm{s}}(\theta)$ and the total form of the curve. The width $\sigma_{\mathrm{s}}$ is mostly defined by the relative amplitude of the modulation of $I_{s}(\theta)$ with respect to the "baseline" further away from $\theta_{\mathrm{B}}$. The solid line superimposed to the data points represents the theoretical $S$ fluorescence curve, calculated according to Eq. 3 for the best matching parameters, $z_{S}=72 \AA$ and $\sigma_{\mathrm{s}}=12 \AA$. It is seen that the unimodal distribution reproduces the experimental data points well. The obtained center of mass position, $z_{S}=72 \AA$ above the solid surface is based on the assumption that all the metal layers are $24 \AA$ thick, as calculated from the reflectivity data (see section 4.5 ), including the uppermost $\mathrm{Al}$ and $\mathrm{Ni}$ layers. One should expect, however, that the terminal layers are at least partially oxidized and therefore significantly thicker $[40,41]$, so that the actual surface is shifted along the $z$-axis. In a previous study in which the surface position had been calibrated on an absolute scale, the center of mass distribution of $S$ in a similar sample at uncontrolled humidity was found at around $58 \AA$ above the surface [31]. In order to be consistent with this value, a thickening of the terminal metal layers of $14 \AA$ by oxidation has to be assumed, which appears plausible. With this definition of the $z$-axis, which will be used in the following, $z_{S}=58 \AA$ is obtained. The corresponding concentration profile is shown in Fig. $5 \mathrm{c}$ as a solid line. The obtained width of the distribution, 
$\sigma_{\mathrm{s}}=12 \AA$, at first sight seems to reflect the topographic roughness of solid substrate $\left(\sigma_{\text {top }} \approx 11 \AA\right.$, see Methods section). In that case the $S$ distribution could be interpreted as a negligibly thin layer ideally following the corrugations of the solid surface. However, the topographic roughness effectively "seen" by SWXF corresponds to mesoscopic in-plane length scales and can thus be significantly smaller than the one probed by AFM on the micrometer scale. Moreover the deformable organic layers do not necessarily fully follow these corrugations. Instead, they can have lower topographic roughness due to their considerable interfacial tension. In both cases $\sigma_{\mathrm{s}}$ would additionally reflect the intrinsic width of the $S$ distribution, also in terms of its bimodal nature. The upper curve in Fig. 5 a (symbols) shows the angle-dependent $\mathrm{K}$ fluorescence, $I_{\mathrm{K}}(\theta)$, under the same low-humidity conditions. It exhibits the essentially identical angle dependence as $I_{s}(\theta)$, and can thus be described satisfactorily with the same parameters (see solid line superimposed to the data points), apart from the trivial scaling parameter $c_{\max }$. In other words, $\mathrm{S}$ and $\mathrm{K}$ distributions almost perfectly overlap, so that the solid line in Fig. $5 \mathrm{c}$ also describes the $\mathrm{K}$ distribution. This co-localization indicates that the counterions are always found in the immediate vicinity of the negatively-charged sulfate groups of SGS.
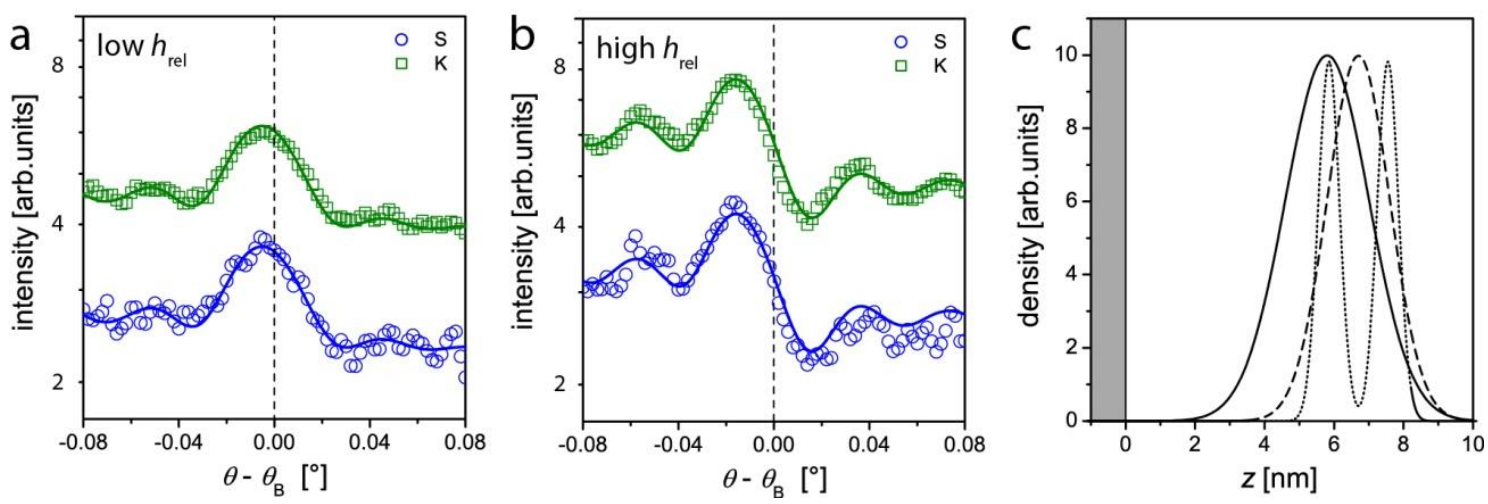

Figure 5: (a) Angle-dependent S and K fluorescence (symbols) from interacting SGS surfaces (see Fig. 1 b) at low humidity. (b) $\mathrm{S}$ and $\mathrm{K}$ fluorescence from the same sample but at high humidity. Solid lines in ( $\mathrm{a}$ and $\mathrm{b}$ ) indicate calculated theoretical intensities corresponding to the best-matching model parameters for the $\mathrm{S}$ distributions at low and high humidity. (c) $\mathrm{S}$ distributions at low humidity (solid line) and at high humidity when assuming unimodal shape (dashed line). The dotted line indicates a bimodal distribution that would result in approximately the same fluorescence signals (see text). The $\mathrm{K}$ distributions at low and high humidity are undistinguishable from the respective $\mathrm{S}$ distributions, so that the curves in panel $\mathrm{c}$ apply to both $\mathrm{S}$ and $\mathrm{K}$.

Fig. $5 \mathrm{~b}$ (bottom curve, symbols) shows $I_{\mathrm{s}}(\theta)$ for interacting SGS surfaces at a high humidity of $h_{\text {rel }} \approx 93 \%$, corresponding to $\Pi \approx 100$ bar (Eq. 2 ). Its angle-dependence is very different from that observed at low humidity, reflecting a significant shift of the $\mathrm{S}$ distribution further away from the solid surface. In fact, the data points for high humidity can still be described satisfactorily with a unimodal distribution (Eq.4), see superimposed solid line for $z_{S}=67 \AA$ and $\sigma_{\mathrm{S}}=9 \AA$. The corresponding concentration profile is shown in Fig. $5 \mathrm{c}$ as a dashed line. This is at first surprising, because the $\mathrm{S}$ distributions belonging to the two sulfur-bearing surfaces are expected to separate for thicker water layers, resulting in a bimodal overall $S$ distribution. The unimodal description however still works, because SWXF is sensitive mainly to the first and second moments of a narrow elemental distribution, $z_{j}=\langle z\rangle$ and $\sigma_{j}^{2}=\left\langle\left(z-z_{j}\right)^{2}\right\rangle$, respectively [31], an aspect that will be discussed 
further below. First we notice that the shift in $z_{\mathrm{S}}$ when going from low to high $h_{\text {rel }}$ is $\delta z=9 \AA$. Assuming that the center of the $S$ distribution belonging to the proximal SGS monolayer is hydrationindependent, the $S$ distribution belonging to the distal monolayer would have to shift by $2 \delta z=18 \AA$ to give the same effect. This extent of swelling upon changing the equivalent pressure from $\Pi \approx 1$ kbar to $\Pi \approx 100$ bar is in rough agreement with the pressure-distance curve of interacting SGS monolayers (Fig. 2). Coming back to the precise shape of the $S$ distribution at high humidity, we note that the $\mathrm{S}$ fluorescence data in Fig. $5 \mathrm{~b}$ are also consistent with a more plausible, bimodal distribution, provided that it exhibits approximately the same first and second moments of the unimodal distribution used to fit the data. As shown in the supporting information, deviations from this approximation are negligible. A symmetrical bimodal distribution, centered around $z_{\mathrm{j}}$ and comprising two Gaussian peaks of individual width $\sigma_{\text {ind, }}$, separated by a distance $\Delta z$, has the same first moment as the unimodal distribution. For the second moment we have $\sigma_{b i \bmod }^{2}=(\Delta z / 2)^{2}+\sigma_{\text {ind }}^{2}$. When $\sigma_{\text {bimod }}$ is set equal to the width $\sigma_{\mathrm{s}}$ obtained for the unimodal distribution, we can solve for $\Delta z$, which is then only a function of $\sigma_{\text {ind }}, \Delta z=2 \sqrt{\sigma_{S}^{2}-\sigma_{\text {ind }}^{2}}$. The dotted line Fig. $5 \mathrm{c}$ shows an exemplary bimodal distribution with compatible first and second moments as obtained for a very low value of the individual peak width, $\sigma_{\text {ind }}=3 \AA$. The upper curve in Fig. $5 \mathrm{~b}$ (symbols) shows the angledependent $K$ fluorescence, $I_{K}(\theta)$, under the same high-humidity conditions. Again, it has the same angle dependence as $I_{S}(\theta)$, indicating perfect overlap of $S$ and $\mathrm{K}$ distributions. This result suggests that, even under highly hydrated conditions the counterions reside closely to the negatively charged surfaces, rather than being evenly distributed in the aqueous phase.

Both the shift in $z_{\mathrm{S}}$ and $z_{\mathrm{K}}$ upon increasing the humidity and the agreement of a bimodal distribution with the experimental data are consistent with the expected swelling behavior of the architecture illustrated in Fig. 1 b. However, it is difficult to rationalize why the second moment of the $S$ distribution does not become larger with increasing hydration level. This behavior may have to do with a more effective healing of the corrugations of the solid surface by the organic layers when they are highly hydrated. In addition one may expect that the saccharide headgroups of SGS undergo significant reorientation with increasing hydration, such that the $S$ atoms get more directed towards the center of the aqueous layer. Alternatively, our experimental results may suggest that the SGS double-monolayers undergo more substantial architectural changes with changing hydration level.

\subsubsection{Interacting lipid-anchored PEG brushes}

Fig. 6 a (bottom curve, symbols) shows the angle-dependent $P$ fluorescence, $I_{\mathrm{P}}(\theta)$, of interacting lipidanchored PEG brushes at a low humidity of $h_{\text {rel }} \approx 40 \%$, corresponding to $\Pi \approx 1.3 \mathrm{kbar}$ (Eq. 2). Even under such dehydrating conditions we expect a clearly bimodal $P$ distribution due to the presence of the PEG layer between the surfaces of the phosphorus-bearing lipid matrix (Fig. $1 \mathrm{c}$ ). Moreover, the maxima of the distribution may exhibit a separation comparable to the SW period above the solid surface $\Lambda=45 \AA$ (due to refraction effects $\Lambda$ is not exactly identical to the multilayer period of $48 \AA$ ). In this case more than one oscillation of the SW contributes to the fluorescence, an effect that imparts some ambiguity to the data. In fact, $I_{\mathrm{P}}(\theta)$ can readily be reproduced with a unimodal Gaussian distribution with $z_{\mathrm{P}}=57 \AA$ and $\sigma_{\mathrm{P}}=10 \AA$, as indicated with the solid line superimposed to the data points. But due to the discrete translational invariance of the $S W$, the same theoretical curve is obtained for a bimodal distribution with an additional, identical Gaussian peak at $z_{\mathrm{p}}{ }^{\prime}=z_{\mathrm{p}}+\Lambda$ 
= $102 \AA$. This bimodal P distribution, which qualitatively is a plausible description of the sample architecture, is shown in Fig. $6 \mathrm{c}$ (upper part, solid line). We remark, however, that on top of this twofold ambiguity there is also another level of ambiguity with respect to details of the individual Gaussian distributions. Namely, the effective width, $\sigma_{\mathrm{p}}=10 \AA$, obtained when assuming a unimodal distribution would also result from a bimodal distribution of sharper peaks that are separated by few $\AA$ more or less than $\Lambda$. However, since the width obtained $\left(\sigma_{P}=10 \AA\right)$ is already rather low, about as low as $\sigma_{\text {top }}$, we assume that this ambiguity is of minor relevance and the $\mathrm{P}$ distribution plotted in Fig. $6 \mathrm{c}$ provides a satisfactory description of the sample structure. This conclusion is also corroborated by the fact that the position of the proximal $P$ peak in the interacting PEG brushes $\left(z_{\mathrm{P}}=57 \AA\right)$ is consistent with the center of the $S$ distribution of the dehydrated SGS double-monolayer $\left(z_{S}=58 \AA\right)$ in light of the similar sample architecture up to the proximal lipid monolayer.

The upper curve in Fig. 6 a (symbols) shows the angle-dependent $S$ fluorescence, $I_{S}(\theta)$, under the same low-humidity conditions. The fluorescence, originating from $S$ atoms in the PDP-labeled endpoints of the PEG chains (Fig. 1 a), assumes a maximum at a significantly larger angle than $I_{P}(\theta)$ in the same plot, reflecting that $\mathrm{P}$ and $\mathrm{S}$ have strongly dissimilar distributions. $I_{\mathrm{S}}(\theta)$ is well reproduced for a slightly broader, unimodal distribution with $\sigma_{\mathrm{S}}=13 \AA$, located between the two peaks of the $P$ distribution. The best match with the data points is achieved with $z_{\mathrm{S}}=88 \AA$, see superimposed solid line. The corresponding $S$ distribution is shown in Fig. 6 c (upper part) as a dashed line. The P and $S$ distributions in Fig. 6 c, representing brush grafting surfaces and polymer endpoints, obviously do not match the expected symmetry of the sample architecture, where $z_{\mathrm{S}}$ would ideally coincide with the center between the two $P$ peaks at $z=80 \AA$. In view of the above-discussed considerable uncertainties in the interpretation of the fluorescence intensities, it is difficult to conclude whether the observed asymmetry is significant, in which case it could reflect, for instance, differences in the packing densities of proximal and distal monolayers.
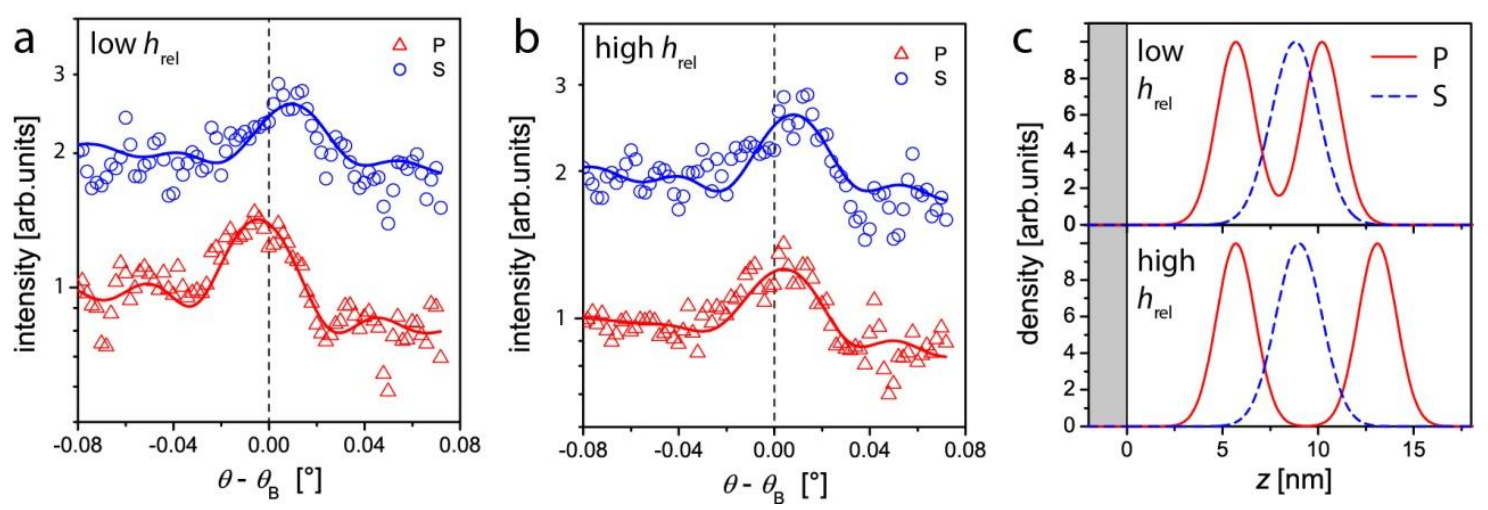

Figure 6: (a) Angle-dependent $P$ and $S$ fluorescence (symbols) from interacting lipid-anchored PEG brushes (see Fig. 1 c) at low humidity. (b) P and S fluorescence from the same sample but at high humidity. Solid lines in (a and $b$ ) indicate calculated theoretical intensities corresponding to the respective model parameters for $\mathrm{P}$ and $\mathrm{S}$ distributions at low and high humidity. (c) P (solid line) and S (dashed line) distributions at low humidity (upper part) and at high humidity (lower part). 
Fig. $6 \mathrm{~b}$ (bottom curve, symbols) shows $I_{\mathrm{P}}(\theta)$ for the interacting PEG brushes at a high humidity of $h_{\text {rel }} \approx 94 \%$, corresponding to $\Pi \approx 80$ bar (Eq. 2). According to the pressure-distance curve in Fig. 2 , swelling by $\gtrsim 2 \mathrm{~nm}$ is expected when reducing the equivalent pressure to this level. A significant change in the $P$ distribution is also evidenced by $I_{P}(\theta)$, in which the feature-to-baseline level is significantly reduced while the maximum is shifted almost exactly to $\theta_{\mathrm{B}}$ at high humidity. An angle dependence like the one obtained for the $P$ fluorescence at high humidity is characteristic for element profiles that are rather equally distributed over the nodes and antinodes of the SW, indicating that the distance between the peaks in a bimodal distribution is closer to an odd than to an even multiple of $\Lambda / 2$. Indeed, as indicated with the solid line superimposed to the data points, $I_{\mathrm{P}}(\theta)$ is well reproduced when the distal peak in the bimodal $\mathrm{P}$ distribution is shifted to $z_{\mathrm{P}}{ }^{\prime}=131 \AA$ by $29 \AA$, which is only a fraction of $\Lambda$. This shift is also roughly consistent with the swelling observed in the pressure-distance curve of the PEG brushes. The corresponding P distribution is shown as a solid line in the lower part of Fig. 6 c. We remark that the experimental data are reproduced even slightly better when the position of the proximal peak is also allowed to move, in which case it moves towards the solid surface by $2 \AA$ (not shown). The upper curve in Fig. $6 \mathrm{~b}$ (symbols) shows the angledependent $S$ fluorescence, $I_{S}(\theta)$, under the same high-humidity conditions. Within the statistical noise of the data points there are no significant differences between $I_{s}(\theta)$ at low and high humidity, respectively. The best-matching unimodal $S$ distribution at high humidity, corresponding to the solid line superimposed to the data points, has $\sigma_{\mathrm{S}}=12 \AA$ and $z_{\mathrm{S}}=90 \AA$, the latter being close to the center between the two P peaks at $z=94 \AA$. The corresponding $\mathrm{S}$ (or end-point) distribution is shown as a dashed line in the bottom part of Fig. 6 c. The $P$ and $S$ distributions suggest that the symmetry of the double-monolayer architecture is recovered at higher humidity. Significant differences in the packing densities of proximal and distal monolayers, as speculated above, are therefore not likely the reason for the asymmetry observed at low humidity. The end-point distribution in a single layer of tethered polymers has previously been determined by SWXF [42]. To our knowledge the present results constitute the first determination of the end-point distribution of confined, interacting polymer brushes.

\section{Discussion and Conclusions}

In the preceding section we have demonstrated that double-monolayer architectures like the ones illustrated in Fig. 1 b and c are well suited for a comprehensive investigation of interacting welldefined surfaces. Namely, such architectures enable the simultaneous determination of pressuredistance curves and of structural details, for example by using $\mathrm{x}$-ray or neutron scattering techniques. We have further demonstrated that SWXF is suited for the label-free and element specific structural investigation of interacting layers. When resolved with high resolution, elemental distributions can yield accurate insight into the molecular configuration of interacting surfaces and its response to changes in the surface separation. In fact, our results suggest that such information is conveniently obtained when elemental distributions are unimodal, for instance when the interacting surfaces have different composition. In many configurations, like the ones described in the present work, the relevant elemental distributions are however multimodal. In these cases detailed molecular conformations are only accessible when the shape of the multimodal distributions can be determined unambiguously. To this end, the topographic roughness of the solid surface poses a limitation, because it leads to a smearing out of the elemental profiles, thus reducing the structural 
detail. Secondly, ambiguity occurs especially when the distances between the distribution maxima are comparable to the SW period. As a consequence, the structural information obtained from the particular set of measurements presented here does not go much beyond what one could also obtain using conventional reflectometry. Nonetheless, our results constitute a proof-of-concept and clearly demonstrate the potential of SWXF when the crucial parameters are optimized: The roughnessinduced smearing out of elemental distributions can be avoided by using multilayer substrates with very smooth surfaces [31]. Ambiguities related to multimodal distributions can be overcome by involving ab-initio information about the sample structure, as well as by a suitable choice of multilayer periods or by working with multiple angle ranges, e.g., in total reflection and with two sufficiently intense Bragg peaks. One can also consider an experiment with similar samples on two $M L$ substrates of different period. Even in the most unfavorable case of a ML period matching exactly the peak distance in a bi-modal distribution the results stay very sensitive to the changes in the key distribution parameters. Finally, $x$-ray reflectivity measurements, carried out in parallel on samples of identical composition but supported by simple solids like sapphire or silicon can yield valuable complementary structural information facilitating the interpretation of the $x$-ray fluorescence data. With that, SWXF may reveal distinct structural aspects not only of generic interactions between soft interfaces but also of specific membrane-membrane interactions for which only the forces have been quantified so far $[43,44]$.

\section{Materials and methods}

\subsection{Materials}

Unless stated otherwise, all chemicals were purchased from Sigma (St. Louis, MO, USA) and used without further purification. Water was purified and double-deionized (MilliQ, Molsheim, France). Sulfoglycolipids (SGS), phospholipids (DSPC), and lipopolymers (PEG-lipid and PDP-PEG-lipid) were purchased from Avanti Polar Lipids (Alabaster, AL, USA). As Illustrated in Fig. 1 a, SGS (3-O-sulfo-Dgalactosyl-ß1-1'-N-heptadecanoyl-D-erythro-sphingosine) has two hydrophobic fatty acid tails and a sulfated galactose monosaccharide headgroup. At neutral $\mathrm{pH}$ the sulfate carries one negative charge. DSPC (1,2-distearoyl-sn-glycero-3-phosphocholine) has two all-saturated $\mathrm{C}_{18}$ chains and a zwitterionic phosphocholine headgroup which carries one $\mathrm{P}$ atom. PEG-lipid (1,2-distearoyl-sn-glycero-3phosphoethanolamine- $\mathrm{N}$-[methoxy(polyethylene glycol)-2000]) is similar to DSPC but a PEG chain comprising 46 monomers is covalently attached to the headgroup. In PDP-PEG-lipid (1,2-distearoylsn-glycero-3-phosphoethanolamine-N-[PDP(polyethylene glycol)-2000]), the terminus of the PEG chain is covalently functionalized with a pyridyldithiopropionate (PDP) moiety containing two $S$ atoms. Multilayer substrates were purchased from X'scitech (Helsinki, Finland) and had 25 repetitions of $\mathrm{Al}(2.4 \mathrm{~nm}) / \mathrm{Ni}(2.4 \mathrm{~nm})$ alternating layers on top of sapphire single crystal wafers with dimensions $8 \mathrm{~mm} \times 8 \mathrm{~mm} \times 1 \mathrm{~mm}$. The terminal Al layer forms amorphous aluminum oxide at the outer surface. Atomic force microscopy (AFM) was used to reveal the topography of the outer surface and in particular its root-mean-square $(\mathrm{rms})$ roughness, $\sigma_{\mathrm{top}} \approx 11 \AA$. Silicon wafers $(150 \mathrm{~mm}$ diameter, $625 \mu \mathrm{m}$ thickness) with a thermal oxide of defined thickness were purchased from SIEGERT Wafer GmbH (Aachen, Germany). 


\subsection{Sample preparation}

Silicon wafers were cut into pieces of $17 \mathrm{~mm} \times 12 \mathrm{~mm}$. Multilayer substrates and silicon substrates were cleaned by washing with chloroform, acetone, ethanol, and water, followed by UV-ozone treatment. The surfaces were then rendered hydrophobic via covalent functionalization with octadecyltrichlorosilane (OTS) by immersion in $1 \mathrm{mM}$ OTS solutions in anhydrous hexadecane for $1 \mathrm{~h}$ and subsequent rinsing in hexadecane and ethanol. SGS, DSPC, and the lipopolymers PEG-lipid and PDP-PEG-lipid were dissolved in chloroform at $2 \mathrm{mg} / \mathrm{mL}$. To obtain DSPC / lipopolymer mixtures at a lipopolymer mole fraction of $10 \mathrm{~mol} \%$, DSPC solution was mixed with PEG-lipid or PDP-PEG-lipid solution, respectively. Double monolayers of SGS or DSPC / lipopolymer on the surfaces multilayer substrates or silicon substrates were prepared using a combination of the Langmuir-Schaefer (LS) and Langmuir-Blodgett (LB) transfer methods [45]. For this purpose, chloroform solutions of SGS or DSPC / lipopolymer mixtures were first spread at the air/water interface in a Langmuir trough (Nima Technology, Coventry, UK; or Riegler \& Kirstein $\mathrm{GmbH}$, Potsdam, Germany) containing $1 \mathrm{mM} \mathrm{KBr}$ aqueous solution (for SGS) or pure water (for DSPC / lipopolymer mixtures). The amphiphilic molecules immobilized at the interface were then compressed to a monolayer with a lateral pressure of $35 \pm 1 \mathrm{mN} / \mathrm{m}$. A first lipid layer was deposited onto the hydrophobically functionalized surfaces via LS transfer, i.e., with the solid surface facing the water surface. This procedure reproducibly yields transfer ratios close to $100 \%$, as evidenced from the negligibly small variation of the surface pressure upon the initial contact and as previously confirmed by neutron reflectometry [19]. In the next step the solid substrate was rotated under water by $90^{\circ}$, so that its surface ended up perpendicular to the water surface. The remaining lipid monolayer at the air/water interface was then removed and replaced with a fresh monolayer at the same lateral pressure. This second monolayer was then transferred on top of the first layer by LB, i.e., by pulling the solid substrate upwards. The LB transfer was confirmed to be effective and reproducible by ellipsometry (see section 4.3. and supporting material).

\subsection{Ellipsometry measurements}

Ellipsometry enables the characterization of interfacial layers in terms of refractive indices and thicknesses. The method is based on the change in the polarization state of light upon reflection from the surface. For a given refractive index $n$, the change depends on the layer thickness and is quantified in terms of the phase difference $\Delta$ and the amplitude ratio $\psi$ encoded in the ratio between the complex reflection coefficients $R_{\mathrm{s}}$ and $R_{\mathrm{p}}$ for $s$ and $p$ polarizations, respectively [46]:

$$
R_{p} / R_{S}=\tan \Psi e^{-i \Delta}
$$

For ellipsometry measurements, silicon chips with thermal oxide were used as substrates for the double monolayers. Silicon has the complex refractive index $n_{\mathrm{si}}=3.885-0.018 \mathrm{i}$ [47]. Measurements were conducted at an incident angle of $70^{\circ}$, with an Optrel Multiskop ellipsometer working with a wavelength $\lambda_{\text {elli }}=632.8 \mathrm{~nm}$. For the known refractive index of $\mathrm{SiO}_{2}, n_{\mathrm{SiO} 2}=1.456$ [48], the obtained measurement values $\left(\Delta=79.3^{\circ}, \Psi=44.3^{\circ}\right)$ correspond to an oxide layer thickness of $105.5 \mathrm{~nm}$, close to the nominal value of $100 \mathrm{~nm}$. In the next step, the measurement values obtained for the fully dehydrated $\left(h_{\text {rel }}<5 \%\right)$ samples were modeled with an additional single layer for the organic film comprising OTS and the two deposited monolayers in order to obtain their thicknesses in the 
absence of water. In this procedure, the refractive index of the organic film was assumed as $n_{\text {org }}=1.46$, a typical value for organic materials [49-51]. The obtained layer thicknesses exhibit excellent proportionality to the nominal material amount deposited, see supporting material. In the last step, the measurement values obtained at controlled humidity were modeled with an additional water layer $\left(n_{\text {wat }}=1.33\right.$ ) while accounting for the known optical parameters of oxide and dry organic layers. In this way, the humidity-dependent equivalent thickness of the water layer $D_{\mathrm{w}}\left(h_{\text {rel }}\right)$ was determined. Humidity was controlled by placing the samples inside a closed chamber through which humidified $\mathrm{N}_{2}$ was streamed. The gas was humidified by letting it pass through a temperaturecontrolled water bath in the form of $\mathrm{mm}$-sized bubbles. High humidities were realized by elevating the water temperature to close to or even slightly above the sample temperature. Low humidities were realized either by lowering the bath temperature or by mixing the humidified $\mathrm{N}_{2}$ stream with dry $\mathrm{N}_{2}$. The humidity at the sample position was measured with a calibrated humidity sensor (SHT75, Sensirion AG, Staefa, Switzerland) measurement uncertainty $\Delta h_{\text {rel }}= \pm 2 \%$ ), placed close to the sample surface. The equivalent dehydrating pressure follows from $h_{\text {rel }}$ according to Eq. 2.

\subsection{X-ray experiments}

X-ray specular reflectometry and SWXF experiments were carried out at the ID10 beamline of ESRF (Grenoble, France). The sample surface was oriented vertically. The beam was focused in vertical direction while its angular divergence was kept low in horizontal direction in order to obtain a nearparallel incident $x$-ray wave in the scattering plane. The latter is required to scan the Bragg reflection condition of the periodic metal multilayers with high angular resolution. The beam diameter was $40 \mu \mathrm{m}$ horizontally and $500 \mu \mathrm{m}$ vertically. For an illumination close to $\theta_{B}=1.13^{\circ}$, the size of the beam footprint on the surface thus was $500 \mu \mathrm{m}$ vertically and $\approx 2 \mathrm{~mm}$ horizontally. The beam energy was $7.0 \mathrm{keV}$, corresponding to a wavelength of $\lambda=1.77 \AA$. The fluorescence radiation was measured using a silicon drift detector (Vortex-EM, SII NanoTechnology) placed perpendicular to the beam direction and about $3 \mathrm{~mm}$ from the surface. To improve measurement statistics without beam damage to the samples, the angular scans were repeated several times with vertically shifted beam position on the sample surface. In fact, when for test purposes the same position was scanned several times, no significant change in the signals was observed, suggesting that beam damage is negligible.

\subsection{Layer representation of the periodic $\mathrm{Al} / \mathrm{Ni}$ layers}

Specular reflectometry from the bare multilayer substrates was measured in order to determine their layered structure, the latter being a prerequisite for accurately modeling the angle dependent SW intensity $\Phi(\theta, z)$. The reflectivity $R(\theta)$ denotes the $\theta$-dependent ratio between the intensities of reflected and incident beams, respectively, and contains information on the electron density distribution perpendicular to an interface. Fig. 7 shows $R(\theta)$ of a multilayer substrate in air, measured in $\theta-2 \theta$ scans with a point detector placed at $2 \theta$ (see Fig. 2 a). The substrate was functionalized with OTS, which owing to its weak electron density contrast (when compared to the solid metal layers) could be safely neglected in the reflectivity analysis. Experimental data (symbols) are corrected for the $\theta$-dependent size of the beam footprint on the sample surface. The red solid line represents the theoretically modeled reflectivity based on a suitable layer representation of the periodic $\mathrm{Al} / \mathrm{Ni}$ layers. It was computed from the layer representation via application of Fresnel's reflection laws at 
each interface and a phase-correct summation using the iterative recipe of Parratt [52]. The model assumes 25 periodic repetitions of alternating $\mathrm{Al}$ and $\mathrm{Ni}$ layers on top of a sapphire $\left(\mathrm{Al}_{2} \mathrm{O}_{3}\right.$, electron density $\left.1.19 \mathrm{e}^{-} / \AA^{3}\right)$. Thickness and electron density of $\mathrm{Al}$ and $\mathrm{Ni}$ layers $\left(d_{\mathrm{Al}}, d_{\mathrm{Ni}}, \rho_{A l}^{e l}\right.$, and $\rho_{N i}^{e l}$, respectively) as well as their interfacial roughness were adjustable fitting parameters. The bestmatching model parameters were obtained in a minimum $\chi^{2}$ fit to the experimental data using the programs Motofit [53] and a web-based tool by Sergey Stepanov for reflectivity analysis [54]. The best match was obtained for $d_{\mathrm{Al}} \approx d_{\mathrm{Ni}} \approx 24 \AA$ and $\rho_{A l}^{e l}=1.23 \mathrm{e}^{-} / \AA^{3}$ and $\rho_{N i}^{e l}=2.09 \mathrm{e}^{-} / \AA^{3}$. All roughness parameters were found to be no larger than $10 \AA$. It should be noted that $\rho_{A l}^{e l}$ as obtained is about $50 \%$ higher than the literature value for pure Al $\left(0.80 \mathrm{e}^{-} / \AA^{3}\right)$, while $\rho_{N i}^{e l}$ is slightly lower (about $9 \%$ ) than the literature value for pure $\mathrm{Ni}\left(2.29 \mathrm{e}^{-} / \AA^{3}\right)$, indicating that significant intermixing between $\mathrm{Al}$ and Ni layers occurs.

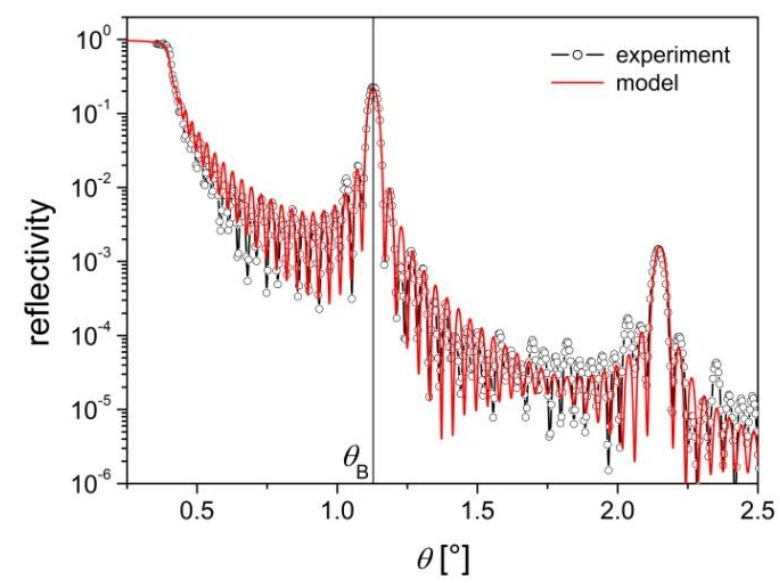

Figure 7: Measured reflectivity curve of a solid multilayer substrate (symbols) together with theoretical reflectivity (solid red line) calculated using a suitable electron density layer model of the periodic $\mathrm{Al} / \mathrm{Ni}$ structure. The vertical straight line indicates the Bragg angle $\theta_{\mathrm{B}}$.

\section{Acknowledgements}

We thank Jean Daillant for insightful comments and Aurelio Barbetta and Anne Heilig for help with sample preparation and complementary AFM measurements, respectively. Beamtime allocation by the ESRF is gratefully acknowledged. This work was financially supported by the Max Planck Society and by the German Research Foundation (DFG) via Emmy-Noether Grant SCHN1396/1.

\section{References}

1. Evans DF, Wennerström H. The Colloidal Domain - Where Physics, Chemistry, Biology, and Technology Meet. New York: VCH, 1994.

2. Mouritsen OG. Life - As a Matter of Fat: Springer, 2005.

3. Schneck E. The Interaction between Soft Interfaces: Forces and Structural Aspects. Advanced Materials Interfaces 2016.

4. Alberts B, Bray D, Lewis J, Raff M, Roberts K, Watson JD. Molecular Biology of the Cell. 4 ed: Garland Science, 2002.

5. Eggens I, Fenderson B, Toyokuni T, Dean B, Stroud M, Hakomori SI. Specific interaction between Lex and Lex determinants. A possible basis for cell recognition in preimplantation embryos and in embryonal carcinoma cells. Journal of Biological Chemistry 1989;264(16):9476-9484. 
6. Rosenhahn A, Schilp S, Kreuzer HJ, Grunze M. The role of "inert" surface chemistry in marine biofouling prevention. Physical Chemistry Chemical Physics 2010;12(17):4275-4286.

7. Webb MS, Green BR. Effects of neutral and anionic lipids on digalactosyldiacylglycerol vesicle aggregation. Biochimica et Biophysica Acta (BBA)-Biomembranes 1990;1030(2):231-237.

8. Lau PCY, Lindhout T, Beveridge TJ, Dutcher JR, Lam JS. Differential lipopolysaccharide core capping leads to quantitative and correlated modifications of mechanical and structural properties in Pseudomonas aeruginosa biofilms. Journal of bacteriology 2009;191(21):6618-6631.

9. Bauer C, Bauduin P, Dufrêche JF, Zemb T, Diat O. Liquid/liquid metal extraction: Phase diagram topology resulting from molecular interactions between extractant, ion, oil and water. The European Physical Journal Special Topics 2012;213(1):225-241.

10. Raviv U, Giasson S, Kampf N, Gohy J-Fo, JÃ@rÃ'me R, Klein J. Lubrication by charged polymers. Nature 2003;425(6954):163-165.

11. Lyklema H. Volume III: Liquid-fluid interfaces. Fundamentals of interface and colloid science 2000;3:iii-v.

12. Derjaguin BV, Churaev NV. Surface Forces. New York: Consultants Bureau, 1987.

13. Parsegian VA, Fuller N, Rand RP. Measured work of deformation and repulsion of lecithin bilayers. Proceedings of the National Academy of Sciences 1979;76(6):2750-2754.

14. Israelachvili JN. Intermolecular and Surface Forces. 2 ed. London: Academic Press Inc., 1991.

15. Wijmans CM, Zhulina EB, Fleer GJ. Effect of free polymer on the structure of a polymer brush and interaction between two polymer brushes. Macromolecules 1994;27(12):3238-3248.

16. Als-Nielsen J, McMorrow D. Elements of Modern X-Ray Physics. Chichester: Wiley, 2001.

17. Daillant J, Gibaud A. X-ray and Neutron Reflectivity: Principles and Applications. Berlin: Springer, 2009.

18. Helm CA, Als-Nielsen J. Phospholipid monolayer density distribution perpendicular to the water surface. A synchrotron x-ray reflectivity study. EPL (Europhysics Letters) 1987;4(6):697.

19. Schneck E, Berts I, Halperin A, Daillant J, Fragneto G. Neutron reflectometry from poly (ethylene-glycol) brushes binding anti-PEG antibodies: Evidence of ternary adsorption. Biomaterials 2015;46:95-104.

20. Majewski J, Kuhl TL, Kjaer K, Gerstenberg MC, Als-Nielsen J, Israelachvili JN, et al. X-ray synchrotron study of packing and protrusions of polymer-lipid monolayers at the air-water interface. Journal of the American Chemical Society 1998;120(7):1469-1473.

21. Kuhl TL, Majewski J, Wong JY, Steinberg S, Leckband DE, Israelachvili JN, et al. A neutron reflectivity study of polymer-modified phospholipid monolayers at the solid-solution interface: polyethylene glycol-lipids on silane-modified substrates. Biophysical journal 1998;75(5):2352-2362.

22. Hamilton WA, Smith GS, Alcantar NA, Majewski J, Toomey RG, Kuhl TL. Determining the density profile of confined polymer brushes with neutron reflectivity. Journal of Polymer Science Part B: Polymer Physics 2004;42(17):3290-3301.

23. Mulder DJ, Kuhl TL. Polymer brushes in restricted geometries. Soft Matter 2010;6(21):54015407.

24. Guenoun P, Schalchli A, Sentenac D, Mays JW, Benattar JJ. Free-standing black films of polymers: a model of charged brushes in interaction. Physical review letters 1995;74(18):3628.

25. Schneck E, Demé B. Structural characterization of soft interfaces by standing-wave fluorescence with $X$-rays and neutrons. Current Opinion in Colloid \& Interface Science 2015;20(4):244-252.

26. Bloch JM, Sansone M, Rondelez F, Peiffer DG, Pincus P, Kim MW, et al. Concentration profile of a dissolved polymer near the air-liquid interface: X-ray fluorescence study. Physical review letters 1985;54(10):1039.

27. Daillant J, Bosio L, Benattar JJ, Blot C. Interaction of cations with a fatty acid monolayer. A grazing incidence $x$-ray fluorescence and reflectivity study. Langmuir 1991;7(4):611-614.

28. Schneck E, Schubert T, Konovalov O, Quinn B, Gutsmann T, Brandenburg K, et al. Quantitative determination of ion distributions in bacterial lipopolysaccharide membranes by grazing-incidence $X$ ray fluorescence. Proc Natl Acad Sci USA 2010;107:9147-9151. 
29. Bedzyk MJ, Bilderback DH, Bommarito GM, Caffrey M, Schildkraut JS. X-ray standing waves: a molecular yardstick for biological membranes. Science 1988;241(4874):1788-1791.

30. Bedzyk MJ, Bommarito GM, Caffrey M, Penner TL. Diffuse-double layer at a membraneaqueous interface measured with x-ray standing waves. Science 1990;248(4951):52-56.

31. Schneck E, Scoppola E, Drnec J, Mocuta C, Felici R, Novikov D, et al. Atom-Scale Depth Localization of Biologically Important Chemical Elements in Molecular Layers. Proc Natl Acad Sci USA 2016;113(34):9521-9526.

32. Demé $B$, Cataye $C$, Block MA, Maréchal E, Jouhet J. Contribution of galactoglycerolipids to the 3-dimensional architecture of thylakoids. The FASEB Journal 2014;28(8):3373-3383.

33. Weinbaum S, Tarbell JM, Damiano ER. The structure and function of the endothelial glycocalyx layer. Annu Rev Biomed Eng 2007;9:121-167.

34. Murat M, Grest GS. Interaction between grafted polymeric brushes: A molecular-dynamics study. Physical review letters 1989;63(10):1074.

35. Sackmann E. Supported membranes: scientific and practical applications. Science 1996;271(5245):43-48.

36. Wei Z, Prescott SW. Scattering approaches to probing surface layers under confinement. Current Opinion in Colloid \& Interface Science 2015;20(4):253-260.

37. Lis L, McAlister M, Fuller N, Rand RP, Parsegian VA. Interactions between neutral phospholipid bilayer membranes. Biophysical journal 1982;37(3):657.

38. Petrache HI, Gouliaev N, Tristram-Nagle S, Zhang R, Suter RM, Nagle JF. Interbilayer interactions from high-resolution x-ray scattering. Physical Review E 1998;57(6):7014.

39. Kenworthy AK, Hristova K, Needham D, McIntosh TJ. Range and magnitude of the steric pressure between bilayers containing phospholipids with covalently attached poly (ethylene glycol). Biophysical journal 1995;68(5):1921.

40. Cerezo A, Petford-Long AK, Larson DJ, Pinitsoontorn S, Singleton EW. The formation mechanism of aluminium oxide tunnel barriers. Journal of materials science 2006;41(23):7843-7852.

41. Vrublevsky I, Parkoun V, Schreckenbach J, Marx G. Effect of the current density on the volume expansion of the deposited thin films of aluminum during porous oxide formation. Applied Surface Science 2003;220(1):51-59.

42. Basu JK, Boulliard JC, Capelle B, Daillant J, Guenoun P, Mays JW, et al. Direct Probe of EndSegment Distribution in Tethered Polymer Chains. Macromolecules 2007;40:6333-6339.

43. Leckband DE, Schmitt FJ, Israelachvili JN, Knoll W. Direct force measurements of specific and nonspecific protein interactions. Biochemistry 1994;33(15):4611-4624.

44. Wong JY, Kuhl TL, Israelachvili JN, Mullah N, Zalipsky S. Direct measurement of a tethered ligand-receptor interaction potential. Science 1997;275(5301):820-822.

45. Tamm L, McConnell HM. Supported phospholipid bilayers. Biophys J 1984;47:105-113.

46. Azzam RMA, Bashara NM. Ellipsometry and polarized light: North-Holland. sole distributors for the USA and Canada, Elsevier Science Publishing Co., Inc., 1987.

47. Adachi S. Model dielectric constants of Si and Ge. Physical Review B 1988;38(18):12966.

48. Malitson $\mathrm{IH}$. Interspecimen Comparison of the Refractive Index of Fused Silica. JOSA 1965;55(10):1205-1209.

49. Wong JE, Rehfeldt F, Hänni P, Tanaka M, Klitzing Rv. Swelling behavior of polyelectrolyte multilayers in saturated water vapor. Macromolecules 2004;37(19):7285-7289.

50. Ruths J, Essler F, Decher G, Riegler H. Polyelectrolytes I: polyanion/polycation multilayers at the air/monolayer/water interface as elements for quantitative polymer adsorption studies and preparation of hetero-superlattices on solid surfaces. Langmuir 2000;16(23):8871-8878.

51. Reiter $\mathrm{R}$, Motschmann $\mathrm{H}$, Knoll W. Ellipsometric characterization of streptavidin binding to biotin-functionalized lipid monolayers at the water/air interface. Langmuir 1993;9(9):2430-2435.

52. Parratt LG. Surface studies of solids by total reflection of X-rays. Physical review 1954;95(2):359.

53. Nelson A. Co-refinement of multiple-contrast neutron/X-ray reflectivity data using MOTOFIT. Journal of Applied Crystallography 2006;39(2):273-276. 
54. Stepanov S, Forrest R. Fitting dynamical X-ray diffraction data over the World Wide Web. Journal of Applied Crystallography 2008;41(5):958-962. 